\title{
Ambiente de Desenvolvimento e Início da Vida Reprodutiva em Mulheres Brasileiras
}

\author{
Environment of Development and the Start of Brazilian Women's \\ Reproductive Life
}

\author{
Eulina da Rocha Lordelo ${ }^{*}, a$, Maria Lucia Seidl-de-Moura ${ }^{b}$, Mauro Luís Vieira $^{c}$, \\ Vera Sílvia Raad Bussab ${ }^{d}$, Angela Donato Oliva ${ }^{b, e}$, Rosana Suemi Tokumaru $^{f}$ \\ \& Regina Célia Souza Britto ${ }^{g}$ \\ ${ }^{a}$ Universidade Federal da Bahia, Salvador, Bahia, \\ ${ }^{b}$ Universidade do Estado do Rio de Janeiro, Rio de Janeiro, Brasil, \\ ${ }^{c}$ Universidade Federal de Santa Catarina, Florianópolis, Brasil, ${ }^{d}$ Universidade de São Paulo, \\ ${ }^{e}$ Universidade Federal do Rio de Janeiro, Rio de Janeiro, Brasil, \\ ${ }^{f}$ Universidade Federal do Espirito Santo, Vitória, Brasil \& ${ }^{g}$ Universidade Federal do Pará, Belém, Brasil
}

\begin{abstract}
Resumo
Modelos teóricos inspirados na teoria da história de vida têm avaliado padrões de reprodução humanos em países desenvolvidos, com resultados ainda não conclusivos. Em vista das condições de vida na população brasileira, foram investigadas relações entre marcos da carreira reprodutiva feminina, condições ambientais e variáveis psicossociais relacionadas às condições de criação. Foram entrevistadas 606 mulheres em seis estados. Os resultados apóiam a teoria da história de vida, mostrando associações entre condições de vida na infância e início da vida sexual e da reprodução, mas não com a idade da menarca. Sugerimos que diferentes marcadores da vida reprodutiva podem estar sob controle de diferentes fenômenos e que a diversidade de condições da população brasileira oferece contextos alternativos para testar hipóteses. Palavras chave: Psicologia Evolucionista; Teoria da História de Vida; Carreira reprodutiva; Menarca.
\end{abstract}

\begin{abstract}
Several models inspired by the life history theory have assessed the patterns of human reproduction in developed countries with promising but inconclusive results. Considering the diversity of life conditions in Brazil, we investigated the relationship between the time of main events in female reproductive life, environmental conditions and psychosocial variables related to child-rearing. We interviewed 606 women in six different states in Brazil. Results corroborate the life history theory, showing associations among the investigated factors, especially between life conditions during childhood and the beginning of sexual life and reproduction, but not to sexual maturity. We suggest that landmarks in reproductive life, although related, can be under the influence of several phenomena. Diversity of life conditions in Brazil offers alternative contexts to hypotheses testing.

Keywords: Evolutionary Psychology; Life History Theory; Reproductive life; Pubertal Timing/Menarche.
\end{abstract}

De acordo com a teoria da história de vida, a maioria dos organismos possui adaptações para ajustar sua vida reprodutiva, conforme as circunstâncias ambientais. Em humanos, a natureza desse ajuste envolve dois trade-offs fundamentais (Kaplan \& Gangestad, 2005): quantidade versus qualidade da prole e reprodução atual versus futura. O primeiro trade-off foi enfatizado por Draper e

*Endereço para correspondência: Universidade Federal da Bahia, Faculdade de Filosofia e Ciências Humanas, Departamento de Psicologia, Rua Estrada de São Lázaro, 197, Federação, Salvador, BA, Brasil, CEP 40210-909. E-mails: eulina@ufba.br, mlseidl@gmail.com, maurolvieira@gmail.com,vsbussab@gmail.com, angeladonatoliva@uol.com.br, tokumaru@usp.bre rcsb@uol.com.br
Harpending (1982); eles propuseram que o ajuste se daria conforme o esforço parental esperado numa dada circunstância: condições de alto stress predizem baixo esforço parental, favorecendo a quantidade, ou seja, mais filhos e menos cuidado parental. Eles focalizaram especificamente a ausência do pai como um sinalizador de stress, direcionando a estratégia reprodutiva mais quantitativa. Na visão de Chisholm (1999), entretanto, o ajuste se daria na tensão entre reprodução atual versus reprodução futura, e estratégias reprodutivas humanas "freqüentemente parecem evolutivamente contingentes a indicadores atuais de risco futuro e incerteza" (Chisholm, 1999, p. 52).

Tomando como base a perspectiva de Draper e Harpending (1982), Belsky, Steinberg e Draper (1991) 
propuseram um modelo de carreira reprodutiva levando em conta as condições de criação, especialmente o clima psicossocial do ambiente familiar, que incluía a idade da menarca como um marcador importante. O modelo assenta-se na premissa de que a idade da menarca foi selecionada para maximizar o sucesso reprodutivo total (Kaplan \& Gangestad, 2005), uma vez que está estreitamente associada ao início da vida reprodutiva, uma associação que tem sido confirmada repetidas vezes (Hoier, 2003; Udry \& Cliquet, 1982).

Amadurecimento sexual, início da vida sexual ativa e a idade do nascimento do primeiro filho são componentes da carreira reprodutiva, passíveis, a princípio, de ajustamento a um padrão particular representando soluções para os trade-offs do ciclo vital. No entanto, não é certo que esses eventos operem uniformemente. Por exemplo, no que diz respeito à idade da menarca, as pesquisas têm abordado, entre outros, fatores ligados à herança genética, raciais, nutricionais, peso e altura, eventos pré-natais e história de amamentação e, mais recentemente, fatores psicossociais, bem como impactos da menarca no desenvolvimento psicossocial da jovem, que constituem linhas de pesquisa diferenciadas (Blell, Pollard, \& Pearce, 2008).

Há fortes evidências de um componente genético individual na determinação da idade da menarca, oriundas de estudos com gêmeos, apontando para um percentual de herdabilidade que varia de 50 a $80 \%$ (Anderson, Duffy, Martin, \& Visscher, 2007; Rowe, 2002; van den Berg \& Boomsma, 2007). Além disso, parece haver também um componente de variabilidade associado à origem ou raça da população. Por exemplo, Danker-Hopfe (1986) encontrou, em populações originárias de diferentes regiões da Europa, vivendo todas na Austrália contemporânea, diferenças significativas nas médias da menarca, após controlar o fator nutrição. Estudos mais recentes realizados em amostras nacionais têm encontrado resultados na mesma direção (Dunbar, Sheeder, Lezzote, Dabelea, \& Stevens-Simons, 2008; Rowe \& Rodgers, 1994; Sun et al., 2002; Wu, Mendola, \& Buck, 2002).

Além dos fatores genéticos e raciais, aspectos da variabilidade ambiental, material e psicossocial, parecem exercer efeitos sobre o fenômeno. O primeiro desses atores, a nutrição, parece ser a mais importante variável causal na idade da menarca (Posner, 2006). Gage, MacCullough, Weitz, Dutt e Abelson (1989) sustentam que nutrição e status de saúde são os principais preditores da idade da menarca, como evidenciado no declínio dessa média durante o século XX na Europa, de 17 para 12 anos e das grandes diferenças entre meninas de países desenvolvidos e em desenvolvimento, variando de 12-13 nos primeiros até 17-18 nos demais. A mesma tendência foi encontrada na China (Graham, Larsen, \& Xu, 1999), na Coréia (Park, Shim, Kim, \& Eun, 1999) e nos Estados Unidos (Graber, Brooks-Gunn, Paikoff, \& Warren, 1994), entre outros.

Na dimensão psicossocial do ambiente, a maioria dos estudos tem se apoiado na teoria do investimento parental de Trivers (1972), propondo que escassez e instabilidade de recursos estarão associadas a uma carreira reprodutiva mais quantitativa e/ou mais precoce, aplicando-se a teoria não só a espécies, mas também a indivíduos de cada espécie. Nessa linha estão os estudos que tomaram como ponto de partida o modelo de Belsky e colaboradores (Belsky et al., 1991; Moffit, Caspi, Belsky, \& Silva, 1992). Eles encontraram associação entre ambiente familiar (ausência do pai e discórdia conjugal) e idade precoce da menarca. Estudo posterior, controlando a idade da menarca das mães, encontrou a mesma associação (Graber et al., 1994). Um padrão similar de resultados, mas enfatizando conflitos no ambiente familiar, particularmente com as mães, foi também encontrado por Kim e Smith (1998), que sugeriram uma possível interação com a transmissão genética da idade da menarca.

Outros estudos têm confirmado os achados, em geral, embora enfatizando diferentes aspectos. Por exemplo, Pettit e Bates (1999), examinaram a qualidade do investimento paternal e encontraram associação positiva entre presença e alto envolvimento do pai nos primeiros anos de vida e menarca mais tardia. Ellis e Garber (2000) identificaram uma trajetória causal entre problemas psicopatológicos da mãe, relações familiares conflituosas, ausência do pai/presença de padrasto e menarca precoce. Eles também encontraram que a ausência do pai e a presença de um padrasto predizem, independentemente, menarca precoce, apontando para diferentes mecanismos regulando o evento. Quinlan (2003) também encontrou, em uma grande amostra retrospectiva dos EUA, associação entre ausência do pai nos primeiros anos de vida e maturação e vida sexual mais precoces, resultados que vem sendo confirmados geralmente (Bogaert, 2008; Vigil \& Geary, 2006). Também Tither e Ellis (2008) identificaram um poderoso efeito mediador da exposição a comportamento paterno severamente disfuncional na antecipação da menarca, de modo que a ausência paterna em si revelou-se bem menos importante do que esperado até então.

Hoier (2003) testou quatro modelos evolucionários, não mutuamente exclusivos, para explicar a idade da menarca e outros indicadores de carreira reprodutiva, em uma amostra de 321 mulheres alemãs de várias idades: (a) modelo de aceleração psicossocial, de Belsky et al. (1991); (b) escassez masculina; (c) interesse reprodutivo maternal e (d) indicação de poliginia. Seus resultados não apoiaram o modelo de Belsky et al. (1991), quanto aos mecanismos desenvolvimentais responsáveis pelos efeitos da ausência do pai em acelerar a menarca, uma vez que esta não mostrou associação com sociosexualidade, a tendência dos indivíduos a adotarem uma orientação com relação a parceiros sexuais mais ou menos restrita (Schmitt, 2005). Ela também encontrou evidências para apoiar os modelos de escassez masculina e o do interesse reprodutivo materno, mas não para o modelo de indicação de poliginia (Kanazawa, 2001). Maestripieri, Roney, DeBias e Spaepen (2004) também encontraram associa- 
ção entre ausência do pai na infância e menarca mais precoce, bem como maior interesse em estímulos visuais relacionados a bebês.

No Brasil, alguns estudos encontraram uma tendência histórica para antecipação da idade da menarca, acompanhando a melhora de condições de vida da população (Picanço, 1995; Tavares et al., 2000; Vitalle, Tomioka, Juliano, \& Amâncio, 2003). Estudos buscando relacionar menarca a condições ecológicas ou psicossociais, entretanto, são raros. Lago (2002), por exemplo, controlou o nível de escolaridade dos pais e encontrou que, embora a antecipação da idade da menarca seja uma tendência secular geral na população, ela é mais acentuada entre as meninas cujos pais têm nível de escolaridade mais baixo. Lordelo et al. (2006), estudando uma amostra de 37 mulheres de um único bairro e mesmo nível socioeconômico em Salvador, encontraram associação entre condições psicossociais de criação e os padrões de carreira reprodutiva, especialmente a idade da primeira união conjugal e do primeiro filho, mas não da menarca.

Essa diversidade e, algumas vezes, incongruência de resultados foi analisada detalhadamente por Ellis (2004), que sintetizou cinco teorias de nível intermediário para explicar a idade da menarca. A primeira delas (teoria energética), sugere que escassez de recursos retardará a reprodução como um todo, começando pela menarca. A segunda teoria, a da supressão por stress, constitui uma extensão da teoria energética e propõe que o aumento dos níveis de stress, físico ou psicossocial, atrasa o desenvolvimento em geral e a menarca em particular. A análise de Ellis distingue estressores físicos e sociais e sugere que eles podem ter efeitos diferentes e até opostos. A aparente inconsistência entre essas duas teorias é discutida por Ellis, que sugere uma possibilidade de conciliá-las, concebendo a reação ao estresse, tanto positiva quanto negativa, como abertura ou permeabilidade à experiência ambiental, desenvolvendo-se essa sensibilidade num formato curvilíneo: ambientes altamente apoiadores ou altamente estressantes gerariam igualmente alta reatividade, de modo a retardar a menarca, enquanto níveis moderados de estresse levariam a menor sensibilidade e reatividade aos eventos do ambiente.

A terceira teoria, a da aceleração psicossocial, caminha na direção oposta, ao sustentar que escassez e/ou imprevisibilidade de recursos estão associados à precocidade do amadurecimento sexual e a um padrão de investimento parental reduzido, sendo os recursos direcionados mais para o esforço reprodutivo e menos para o investimento parental, gerando um padrão mais instável nos relacionamentos românticos ou conjugais. A quarta teoria, a do investimento paternal, realça o papel do pai na família e as atitudes e comportamentos maternos em relação a homens como fatores relevantes na avaliação infantil das configurações ambientais durante a infância que balizam os ritmos do ciclo vital. Finalmente, a teoria do desenvolvimento da criança sugere que a função da puberdade é fazer a transição entre o período não reprodutivo e o reprodutivo, sendo a avaliação do ambiente da infância usada para ajustar o ciclo vital, reduzindo ou aumentando o período de crescimento e aquisição de habilidades. Essa teoria é compatível com os modelos de investimento parental e aceleração psicossocial quanto ao papel das experiências infantis em acelerar a maturidade sexual e a reprodução, sem implicar efeitos na sociossexualidade, como na teoria do investimento parental.

Mais recentemente, Belsky et al. (2007) realizaram um estudo longitudinal com 756 crianças brancas dos Estados Unidos, incluindo meninos, usando medidas repetidas de amadurecimento sexual e ambiente familiar, controlando a idade da menarca da mãe e sensibilidade da criança à estimulação positiva e negativa. Eles encontraram que estilos parentais negativos foram associados a amadurecimento sexual mais precoce, mas apenas entre as meninas, uma associação mais forte entre aquelas que foram avaliadas como altas em reatividade negativa na infância. No entanto, a idade da menarca da mãe foi um preditor mais forte da menarca da menina do que as condições de criação.

Também Ellis e Essex (2007) investigaram longitudinalmente uma amostra de 120 meninos e meninas, começando por avaliações de qualidade do ambiente familiar durante a pré-escola e, aos sete anos de idade, dos níveis hormonais. Com 11 anos, foram coletadas informações sobre características sexuais secundárias. Os resultados mostraram que alto investimento parental, incluindo pais e mães, foi um preditor confiável de amadurecimento sexual mais tardio. Baixo índice de massa corporal, menarca tardia da mãe e nível socioeconômico mais alto também foram preditores independentes de retardo na menarca, mas a associação entre investimento parental de alta qualidade na infância e menarca tardia permaneceu estatisticamente significante, mesmo após o controle dessas outras variáveis. Os resultados foram vistos como compatíveis com as teorias da aceleração psicossocial e do desenvolvimento da criança.

Apesar de serem observadas certas tendências comuns a vários dos estudos citados, muitas questões estão em aberto, permanecendo uma grande lacuna no campo quanto a um modelo capaz de traduzir teorias mais amplas sobre investimento parental e história de vida - em componentes específicos para o caso humano.

Além dos aspectos conceituais, assinala-se a baixa diversidade das amostras estudadas. Praticamente todos os estudos contemporâneos que investigaram o assunto têm empregado indicadores de recursos ambientais de natureza psicossocial, basicamente ausência paterna, relacionamento com os pais e atmosfera familiar; em alguns casos, tem sido investigada também a disponibilidade de homens como possíveis parceiros. Virtualmente todas as sociedades de onde foram retiradas amostras podem ser caracterizadas como sociedades afluentes, com abundância de recursos básicos de sobrevivência, e com popula- 
ções com baixa mestiçagem, como é o caso dos estudos de Moffit et al. (1992), Ellis e Garber (2000), Pettit e Bates (1999) e Hoier (2003), realizados nos Estados Unidos, Nova Zelândia e Alemanha.

Qual seria o timing do início da vida reprodutiva em uma população com grande variabilidade, seja em recursos, seja em origem racial? Tendo em vista os diversos modelos teóricos em discussão, e as características da população brasileira, o presente estudo teve como objetivos: (a) avaliar os principais marcos temporais da vida reprodutiva de uma amostra de mulheres brasileiras, especificamente idades da menarca, da primeira relação sexual e do primeiro filho, relacionando-os ao seu ambiente de desenvolvimento e às suas condições atuais de vida; (b) confrontar esses resultados com alguns dos modelos teóricos propostos: energético, aceleração psicossocial e desenvolvimento da criança, avaliando sua pertinência a essas condições particulares.

\section{Método}

\section{Participantes}

Participaram da pesquisa 606 mulheres maiores de 20 anos, selecionadas por acessibilidade, mães de pelo menos uma criança com idade entre 0 e 6 anos de idade, em seis Estados do Brasil, um de cada região geográfica: Pará, Bahia, Espírito Santo, Rio de Janeiro, São Paulo e Santa Catarina. Em cada estado foram estudados dois grupos, um da capital e outro de uma pequena cidade do interior, com menos de 20.000 habitantes, e distante mais de 200 $\mathrm{km}$ da capital do Estado.

Indicadores socioeconômicos sugerem fortes diferenças entre os dois tipos de contextos de onde foram extraídas as amostras. Em 2003, as seis capitais de estado tinham média de PIB per capita (US\$ 16.396) cerca de duas vezes maior que as cidades do interior, onde a média era de US\$ 7.588 [Instituto Brasileiro de Geografia e Estatística (IBGE), 2009b]. Entretanto, a proporção entre homens e mulheres acima de 10 anos em 2000 (IBGE, 2009a) é deficitária nas capitais, sugerindo o efeito da violência dos contextos urbanos (médias de 90,2 homens por 100 mulheres nas capitais e 102,0 no interior). De fato, as taxas de homicídios [Núcleo de Estudos da Violência da Universidade de São Paulo \& Comissão Teotônio Vilela de Direitos Humanos (NEV-USP \& CTV), 2007] nas capitais brasileiras são várias vezes mais altas do que as taxas distribuídas para todo o estado correspondente. As duas subamostras são também diferenciadas em idade (médias de 20,7 nas capitais e 28,4 no interior); em anos de escolaridade (médias de 13,4 nas capitais e 8,9 no interior); e renda (médias de $\mathrm{R} \$ 3057$ nas capitais e $\mathrm{R} \$ 868$ no interior).

\section{Instrumento}

Foi utilizado um questionário geral da pesquisa "Investimento e cuidado parentais: Aspectos biológicos, ecológicos e culturais", que coletou informações sobre uma variedade de aspectos. Foram utilizadas as partes do questionário relativas à situação socioeconômica atual, à história de vida (condições materiais e psicossociais de criação) e à carreira reprodutiva. Para o primeiro grupo de variáveis, foram perguntadas idade, escolaridade em anos e renda familiar total em reais. No segundo grupo, foram coletadas informações sobre local de criação (se rural ou urbano e tamanho da cidade), ambiente familiar (composição familiar e ambiente psicossocial), número de irmãos e ordem de nascimento. No terceiro grupo, foram investigadas as idades, em anos completos, da menarca, da primeira relação sexual e do primeiro filho. A partir das perguntas sobre condições materiais e psicossociais na infância, foi composta uma variável global para estresse na infância, somando-se as respostas aos itens: condições materiais, clima familiar, conflitos com o pai, conflitos com a mãe, conflitos entre os cônjuges, atitudes do pai (e da mãe) em relação à participante, ocorrência de morte de um dos pais na infância e separação dos pais na infância. A medida resultante foi um escore que poderia variar de 8 a 50 (encontrados mínimo de 8 e máximo de 38 ), convertido posteriormente, a partir da análise de frequiências, em três categorias de stress: baixo, médio e alto.

\section{Procedimentos de Coleta e Análise de Dados}

O projeto de pesquisa foi previamente submetido e aprovado pelos comitês de ética em pesquisa das instituições que participaram do estudo. $\mathrm{O}$ recrutamento de participantes foi realizado com o auxílio de agentes de saúde, no interior e por líderes comunitários, nas capitais. Todas as mulheres receberam explicações sobre a pesquisa e foram convidadas a participar, mediante a assinatura de Termo de Consentimento, preservando-se o direito à recusa, o que ocorreu em menos de $10 \%$ dos casos. As entrevistas foram realizadas por pesquisadores previamente treinados, em uma única sessão, nas residências das participantes.

Testes de GLM univariados foram realizados utilizando-se como fatores as diversas variáveis hipotetizadas como preditoras da idade da menarca, da primeira relação e do primeiro filho. Para cada teste de cada uma das VDs, $p<0,01$ foi utilizado, segundo correção de Bonferroni (Kutner, Nachtsheim, Neter, \& Li, 2005).

\section{Resultados}

\section{Variáveis Sociodemográficas}

Conforme pode ser visto na Tabela 1 , mulheres vivendo nas capitais ou em cidades do interior diferenciam-se em idade da menarca $[F(1,598)=6,64 ; p<0,01 ;$ Power $=0,73]$ e do nascimento do primeiro filho $[F(1,602)=$ $27,13 ; p<0,01 ;$ Power $=0,99]$, mas não no início da vida sexual ativa. Particularmente o nascimento do primeiro filho ocorreu bem mais cedo no interior $(21,96$ contra 24,35 nas capitais). 
Tabela 1

Idade da Menarca, Idade da Primeira Relação Sexual e Nascimento do Primeiro Filho Conforme Idade, Contexto Atual, Renda e Escolaridade

\begin{tabular}{|c|c|c|c|c|c|c|c|}
\hline & $N$ & Média $(D P)$ & $F$ & Média $(D P)$ & $F$ & Média $(D P)$ & $F$ \\
\hline \multicolumn{8}{|l|}{ Idade } \\
\hline 20 a 24 anos & 165 & $12,47(1,5)$ & \multirow{4}{*}{0,24} & $16,18(2,2)$ & \multirow[t]{4}{*}{$43,64 * * *$} & $18,53(2,5)$ & \multirow[t]{4}{*}{$142,09 * * *$} \\
\hline 25 a 29 anos & 165 & $12,48(1,5)$ & & $17,36(2,4)$ & & $21,59(3,4)$ & \\
\hline 30 a 34 anos & 136 & $12,55(1,6)$ & & $18,63(3,0)$ & & $25,29(4,6)$ & \\
\hline 35 ou mais anos & 138 & $12,60(1,4)$ & & $19,87(4,0)$ & & $28,39(6,2)$ & \\
\hline \multicolumn{8}{|l|}{ Contexto atual } \\
\hline Capital & 298 & $12,36(1,5)$ & \multirow[t]{2}{*}{$6,64^{*}$} & $17,95(3,0)$ & \multirow[t]{2}{*}{0,21} & $24,35(5,9)$ & \multirow[t]{2}{*}{$27,13 * * *$} \\
\hline Interior & 306 & $12,67(1,5)$ & & $17,83(3,5)$ & & $21,96(5,4)$ & \\
\hline \multicolumn{8}{|l|}{ Renda atual } \\
\hline Até $\mathrm{R} \$ 500,00$ & 136 & $12,56(1,5)$ & \multirow{3}{*}{0,79} & $17,30(3,1)$ & \multirow[t]{3}{*}{$8,00 * * *$} & $20,53(5,1)$ & \multirow[t]{3}{*}{$56,08 * * *$} \\
\hline $\mathrm{R} \$ 501,00$ a $1.800,00$ & 277 & $12,60(1,5)$ & & $17,68(3,2)$ & & $22,32(5,4)$ & \\
\hline 1.801 ou mais & 175 & $12,42(1,5)$ & & $18,68(3,3)$ & & $26,56(5,4)$ & \\
\hline \multicolumn{8}{|l|}{ Escolaridade } \\
\hline Fundamental incompleto & 138 & $12,54(1,6)$ & \multirow{4}{*}{1,90} & $16,92(3,1)$ & \multirow[t]{4}{*}{$14,22 * * *$} & $20,25(4,8)$ & \multirow[t]{4}{*}{$69,85^{* * *}$} \\
\hline $\begin{array}{l}\text { Fundamental completo } \\
\text { e médio incompleto } \\
\text { Segundo grau completo }\end{array}$ & 109 & $12,75(1,5)$ & & $17,33(2,8)$ & & $20,59(4,9)$ & \\
\hline $\begin{array}{l}\text { Segundo grau completo } \\
\text { e superior incompleto }\end{array}$ & 216 & $12,53(1,4)$ & & $17,90(2,8)$ & & $23,15(4,9)$ & \\
\hline $\begin{array}{l}\text { Superior completo } \\
\text { e pós-graduação }\end{array}$ & 137 & $12,30(1,3)$ & & $19,28(3,8)$ & & $28,05(5,1)$ & \\
\hline
\end{tabular}

Nota. ${ }^{*} p<0,05 ; * * p<0,01 ; * * * p<0,001$.

A renda familiar constituiu-se em um fator significativo para diferenciar a idade da primeira relação $[F(2,583)$ $=8,00 ; p<0,01 ;$ Power $=0,96]$, e do nascimento do primeiro filho $[F(2,583)=56,08 ; p<0,01 ;$ Power $=1]$. Ambos os eventos foram mais tardios para mulheres com renda atual mais alta. A média de idade da primeira relação neste caso foi de 18, $68(D P=3,3)$, em contraste com uma média de 17,30 $(D P=3,1)$ para mães do grupo de renda mais baixa. Quanto ao nascimento do primeiro filho, deu-se em média aos 26,56 anos $(D P=5,4)$ para mães do grupo de renda mais alta e aos $20,53(D P=5,17)$ para as do grupo de mais baixa renda. $O$ mesmo não ocorreu em relação à idade da menarca, em que o fator renda não é significativo. No caso das relações estatisticamente significativas observadas, é evidente que a renda atual mais alta não é um verdadeiro preditor de eventos que ocorreram no passado, no entanto, é razoável assumir que mulheres atualmente mais pobres tenham sido mais pobres também no passado, com piores condições de vida.

Em relação à escolaridade, as tendências foram similares. Não houve diferenças estatisticamente significativas entre os grupos quanto à idade da menarca, mas foram observados efeitos significativos para este fator em relação à idade da primeira relação sexual $[F(3,587)=14,22$, $p<0,01 ;$ Power $=1]$ e à idade em que nasceu o primeiro filho $[F(2,583)=69,85 ; p<0,01 ;$ Power $=1]$. Obser- vou-se que mães com o nível mais alto de escolaridade (superior completo pelo menos) tiveram sua primeira relação sexual em média com 19,28 anos $(D P=3,8)$ e que isso aconteceu mais cedo no grupo com o menor nível de escolaridade (menos do que ensino fundamental). Nesse último caso, a média foi de 16,93 anos $(D P=3,1)$.

Finalmente, pode-se pensar no efeito da idade atual da respondente sobre as três variáveis que vêm sendo consideradas. Da mesma forma que nos dois fatores antes discutidos (escolaridade e renda), não há um efeito significativo para a idade da menarca. Um efeito significativo, no entanto, foi observado em relação à idade da primeira relação sexual $[F(3,593)=43,64, p<0,01$; Power $=1]$. Mulheres mais velhas atualmente tiveram sua primeira relação mais tarde $(M=19,87 ; D P=4,0)$, e mulheres mais jovens, com menos de 24 anos, a tiveram mais cedo $(M=16,18 ; D P=2,2)$. Há também um efeito desta variável na idade do nascimento do primeiro filho $[F(3,600)$ $=142,09, p<0,01 ;$ Power $=1]$. Mães mais novas, entre 20 e 24 anos, tiveram seus filhos mais jovens $(M=18,53$, $D P=2,5)$ e mães com mais de 35 anos na ocasião da pesquisa tiveram seus filhos em média mais tarde $(M=$ $28,39$; $D P=6,7)$. Embora uma tendência à iniciação sexual mais precoce venha sendo apontada na literatura, o efeito aqui encontrado é também, provavelmente, resultado dos critérios de escolha da amostra, uma vez que a 
Lordelo, E. R., Seidl-de-Moura, M. L., Vieira, M. L., Bussab, V. S. R., Oliva, A. D., Tokumaru, R. S. \& Britto, R. C. S. (2011). Ambiente de Desenvolvimento e Início da Vida Reprodutiva em Mulheres Brasileiras.

exigência de filhos entre 0 e 6 anos torna provável que mulheres mais velhas que atendam a essa condição tenham tido também sua iniciação sexual mais tardia.

\section{Condições Materiais de Criação}

A idade da menarca foi afetada pelas condições de criação da mulher. Pessoas criadas no interior $(M=12,79$; $D P=1,5)$, em cidades pequenas $(M=12,89 ; D P=1,4) \mathrm{e}$ com muitos irmãos, tiveram menarca mais tardia do que suas contrapartes. Os resultados dos testes GLM para essas variáveis de interesse foram, respectivamente: para local de criação $[F(1,598)=12,16, p<0,01 ;$ Power $=$ 0,94 ; para tamanho da cidade $[F(2,591)=14,17, p<0,01$ Power $=0,99$; e para número de irmãos $[F(3,596)=5,05$, $p<0,01 ;$ Power $=0,92]$, conforme pode ser visto na Tabela 2.

Tabela 2

Idade da Menarca, Idade da Primeira Relação Sexual e Nascimento do Primeiro Filho Conforme Contexto de Criação

\begin{tabular}{|c|c|c|c|c|c|c|c|}
\hline & \multirow[b]{2}{*}{$N$} & \multicolumn{2}{|c|}{ Menarca } & \multicolumn{2}{|c|}{ Primeira relação sexual } & \multicolumn{2}{|c|}{ Primeiro filho } \\
\hline & & Média $(D P)$ & $F$ & Média $(D P)$ & $F$ & Média $(D P)$ & $F$ \\
\hline \multicolumn{8}{|c|}{ Contexto de criação } \\
\hline Capital & 370 & $12,35(1,4)$ & $12,16^{* * *}$ & $17,95(3,4)$ & 0,52 & $23,85(5,9)$ & $15,27 * * *$ \\
\hline Interior & 230 & $12,79(1,5)$ & & $17,77(3,1)$ & & $21,98(5,3)$ & \\
\hline \multicolumn{8}{|c|}{ Tamanho da cidade } \\
\hline Pequena & 250 & $12,89(1,4)$ & $14,17 * * *$ & $17,94(3,5)$ & 0,23 & $22,40(5,6)$ & $7,67 * * *$ \\
\hline Média & 137 & $12,15(1,6)$ & & $17,72(3,1)$ & & $22,51(5,4)$ & \\
\hline Grande & 211 & $12,33(1,4)$ & & $17,94(3,1)$ & & $24,35(6,0)$ & \\
\hline \multicolumn{8}{|l|}{ Número de irmãos } \\
\hline Até 1 irmão & 92 & $12,21(1,3)$ & $5,05^{* *}$ & $17,69(3,7)$ & 1,22 & $24,58(6,0)$ & $2,58^{*}$ \\
\hline 2 ou 3 irmãos & 226 & $12,44(1,4)$ & & $17,78(2,8)$ & & $22,89(5,4)$ & \\
\hline 4 ou 5 irmãos & 129 & $12,43(1,4)$ & & $18,38(3,5)$ & & $23,24(5,9)$ & \\
\hline 6 ou mais irmãos & 157 & $12,90(1,7)$ & & $17,76(3,4)$ & & $22,57(6,0)$ & \\
\hline
\end{tabular}

Nota. $* p<0,05 ; * * p<0,01 ; * * * p<0,001$.

No entanto, esses efeitos estiveram ausentes para a idade da primeira relação, voltando a aparecer no nascimento do primeiro filho. Neste caso, em relação ao fator local de criação, mães que foram criadas em cidades tiveram o primeiro filho mais tarde do que mães criadas no interior $[F(1,602)=15,27 ; p<0,001 ;$ Power $=0,97]$. Em relação ao tamanho da cidade em que foram criadas, as mulheres criadas em cidades pequenas tiveram filhos mais cedo do que aquelas criadas em cidades grandes $[F(2,595)=$ $7,67, p<0,001 ;$ Power $=0,95]$. Finalmente, em relação ao número de irmãos, não houve efeito significativo para $p<0,01$.

Em síntese, no caso do contexto de criação urbano ou rural e do tamanho da cidade, a idade da menarca foi mais precoce em cidades grandes e em pessoas com menos irmãos. No entanto, o padrão referente ao nascimento do primeiro filho foi justamente o oposto: mulheres criadas no interior, em especial cidades pequenas, tiveram o primeiro filho mais tarde. As variáveis de interesse apresentaram padrões de associação similares nos dois contextos: a idade atual da mãe que vive em cidades correlacionou-se com número de irmãos, com a idade da primeira relação e a idade em que nasceu seu primeiro filho. No caso de mães que vivem atualmente no interior, a idade correlacionou-se com as mesmas variáveis, com exceção do número de irmãos. A idade da menarca, no caso de mães que vivem atualmente em cidades, somente se correlacionou com a idade da primeira relação sexual. Para as mulheres que foram criadas no interior, além dessa relação, foi encontrada correlação positiva entre menarca e idade em que nasceu o primeiro filho. Finalmente, a idade da primeira relação, além das associações já relatadas, correlacionou-se com a idade do nascimento do primeiro filho, nos dois contextos. Das pessoas que vivem atualmente em capitais, $87 \%$ foram criadas nesse mesmo ambiente. Dentre as que vivem atualmente no interior, $83 \%$ foram criadas também nesse contexto. Ou seja, as correlações relatadas não seriam muito diversas em relação ao ambiente de criação.

\section{Condições Psicossociais de Criação}

Nenhuma das variáveis psicossociais relacionadas à criação mostrou efeito na idade da menarca (Tabela 3). No entanto, a idade da primeira relação sexual apresentou efeitos do tipo de cuidador até sete anos $[F(2,557)=$ $4,97, p<0,01 ;$ Power $=0,81]$; de sete a 11 anos $[F(2,543)$ $=9,49, p<0,01$; Power $=0,98]$; e depois de 11 anos $[F(2,528)=9,0, p<0,01 ;$ Power $=0,97]$, mas não do nível de estresse percebido na vida familiar pela respondente (alto, baixo ou médio). 
Tabela 3

Médias e Desvios-Padrão da Idade da Menarca, da Primeira Relação Sexual e do Nascimento do Primeiro Filho Segundo Variáveis de Criação

\begin{tabular}{|c|c|c|c|c|}
\hline & & Menarca & $\begin{array}{l}\text { Primeira relação } \\
\text { sexual }\end{array}$ & $\begin{array}{l}\text { Nascimento do } \\
\text { primeiro filho }\end{array}$ \\
\hline & $N$ & $M(D P)$ & $M(D P)$ & $M(D P)$ \\
\hline \multicolumn{5}{|c|}{ Principal cuidador entre zero sete anos } \\
\hline Pai e mãe & 373 & $12,52(1,46)$ & $18,20(3,46)$ & $23,89(5,88)$ \\
\hline Pai ou mãe & 144 & $12,46(1,5)$ & $17,41(2,78)$ & $22,32(5,46)$ \\
\hline Avós & 46 & $12,76(1,48)$ & $17,00(3,01)$ & $20,38(4,9)$ \\
\hline $\mathrm{F}$ & & 0,74 & $4,97 * *$ & $10,24 * * *$ \\
\hline \multicolumn{5}{|c|}{ Principal cuidador entre sete e 11 anos } \\
\hline Pai e mãe & 337 & $12,54(1,4)$ & $18,35(3,49)$ & $24,09(5,9)$ \\
\hline Pai ou mãe & 168 & $12,48(1,5)$ & $17,24(2,9)$ & $21,82(5,46)$ \\
\hline Avós & 41 & $12,54(1,6)$ & $16,71(2,3)$ & $21,34(5,20)$ \\
\hline $\mathrm{F}$ & & 0,10 & $9,49 * * *$ & $11,196 * * *$ \\
\hline \multicolumn{5}{|c|}{ Principal cuidador a partir de 11 anos } \\
\hline Pai e mãe & 320 & $12,55(1,41)$ & $18,37(3,48)$ & $24,16(5,8)$ \\
\hline Pai ou mãe & 184 & $12,46(1,55)$ & $17,22(2,92)$ & $21,85(5,52)$ \\
\hline Avós & 30 & $12,67(1,52)$ & $16,88(2,31)$ & $21,38(5,5)$ \\
\hline $\mathrm{F}$ & & 0,36 & $9,00 * * *$ & $11,36 * * *$ \\
\hline \multicolumn{5}{|l|}{ Estresse percebido } \\
\hline Baixo & 173 & $13,32(1,42)$ & $18,34(3,32)$ & $24,33(5,5)$ \\
\hline Médio & 199 & $12,55(1,44)$ & $18,08(3,45)$ & $23,59(6,14)$ \\
\hline Alto & 164 & $12,64(1,57)$ & $17,43(3,0)$ & $21,96(5,83)$ \\
\hline $\mathrm{F}$ & & 2,12 & $3,44 *$ & $7,48 * *$ \\
\hline
\end{tabular}

Nota. ${ }^{*} p<0,05 ; * * p<0,01 ; * * * p<0,001$.

A idade de nascimento do primeiro filho foi também afetada por essas condições, na direção prevista pelo modelo Belsky. Foram estatisticamente significativos os efeitos do tipo de cuidador nas três faixas etárias e também do nível de estresse percebido pela respondente: tipo de cuidador até sete anos $[F(2,564)=10,24, p<0,01$; Power $=0,99]$; do de sete a 11 anos $[F(2,550)=11,20, p$ $<0,01 ;$ Power $=0,99$ ]; e do principal cuidador depois de 11 anos $[F(2,535)=11,36, p<0,01$; Power $=0,99]$. Nesse caso, também foi observado um efeito significativo para o nível de estresse percebido $[F(2,543)=7,48, p<$ $0,01 ;$ Power $=0,94]$

As idades da primeira relação sexual e do nascimento do primeiro filho ocorreram em média mais cedo, conforme a criação se deu por avós ou pela mãe/pai apenas, repetindo-se o padrão nas três faixas etárias consideradas. No caso da idade do nascimento do primogênito, esta se mostrou também influenciada pelo nível percebido de estresse durante os anos da infância. A Tabela 3 apresenta as médias e desvios-padrão das idades da menarca, da primeira relação e do nascimento do primeiro filho para cada um dos níveis das variáveis consideradas.

\section{Discussão}

Em relação à maturidade sexual, expressa na idade da menarca, encontramos relação com local de criação, ta- manho da cidade em que a mulher foi criada e número de irmãos: mulheres residentes e/ou criadas no interior, em cidades pequenas, e com maior número de irmãos tiveram a menarca mais tardia do que suas contrapartes, mesmo quando a associação entre contexto e tamanho das famílias foi controlada. Outras condições materiais e psicossociais de criação não afetaram a idade da menarca, contrariando parte das previsões da teoria energética e de desenvolvimento da criança. A relação encontrada entre número de irmãos e menarca mais tardia é compatível com a hipótese do interesse reprodutivo maternal (Hoier, 2003): a existência de irmãos mais jovens retardaria a menarca em benefício do apoio no cuidado aos irmãos mais jovens.

Em relação à primeira relação sexual, os resultados apóiam plenamente o modelo de Belsky e colaboradores (Belsky et al., 1991; Moffit et al., 1992), quanto à relação entre escassez ou imprevisibilidade de recursos, ambiente familiar instável ou conflituoso e um início mais precoce da vida sexual.

Finalmente, em relação à idade da mãe no nascimento do primeiro filho, encontrou-se uma tendência à precocidade associada às seguintes condições: mulheres mais jovens (portanto, de geração mais recente), residindo em cidades do interior, criadas no interior e em cidades pequenas, mais pobres e com menor escolaridade, e criadas em ambientes familiares sem a presença dos dois pais 
(criadas por um dos pais apenas ou por avós), e com alto stress percebido durante a infância. Esses achados são congruentes com o modelo de Belsky e também podem ser interpretados à luz da teoria do desenvolvimento da criança, que concebe a menarca como parte da estratégia geral de prolongar ou encurtar o período da infância (Ellis, 2004), em resposta a ambientes mais ou menos confiáveis em termos de recursos.

Esses resultados sugerem que os diferentes eventos marcadores da vida reprodutiva, embora relacionados, podem estar sob controle de diferentes fenômenos. Enquanto o início da vida sexual ativa e da reprodução ocorre consistentemente mais cedo em mulheres que vivem em ambientes mais pobres, em termos de recursos materiais e psicossociais, alinhando-se esses resultados ao modelo de Belsky, outras variáveis podem ser mais importantes em condicionar a maturidade sexual, expressa na idade da menarca. A despeito das correlações encontradas entre idade da menarca, da primeira relação sexual e do primeiro filho, a própria maturidade sexual pode estar sob controle de outras condições, como nutrição e origem racial.

Esses resultados são congruentes com a perspectiva de Ellis (2004) sobre as diversas teorias com relação aos determinantes da idade da menarca, no que diz respeito à complexidade e multideterminação do fenômeno. Nesse caso, a chamada teoria energética (Ellis, 2004) pode ser útil em explicar por que as mulheres criadas no interior tiveram menarca mais tardia, enquanto os demais indicadores são mais precoces, se aceitarmos a suposição razoável de que as condições de nutrição no interior têm sido piores do que as encontradas nas capitais; essa suposição apóia-se nas grandes diferenças em todos os indicadores socioeconômicos existentes entre os dois tipos de contexto (PIB per capita, esperança de vida ao nascer, taxa de alfabetização, mortalidade infantil e IDH). Além disso, é importante lembrar que as capitais brasileiras oferecem aos seus habitantes mais recursos materiais, em termos de educação, trabalho e saúde, mas, ao mesmo tempo, impõem experiências de violência muito elevada.

Essa complexidade resulta, provavelmente, em graves dificuldades para conceituar e mensurar stress material ou psicossocial. As evidências obtidas neste estudo mostram que os contextos do interior são mais pobres, têm uma proporção mais alta de homens por mulheres e são menos violentos em termos de criminalidade. Enquanto a primeira evidência sugeriria menarca mais precoce, as demais levariam ao inverso, menarca mais tardia. No entanto, o fato de que a iniciação sexual e a primeira gravidez ocorrem mais cedo nesses contextos sugere que apenas a idade da menarca não se conforma às predições do modelo Belsky. Aqui, provavelmente, intervêm outros fatores, desconhecidos até agora.

\section{Considerações Finais}

O estudo aqui relatado produziu as primeiras evidências na população brasileira sobre as relações entre carreira reprodutiva e antecedentes psicossociais, numa perspectiva evolucionária, em concordância com os resultados obtidos por Lordelo et al. (2006) e outros pesquisadores (Lago, 2002; Picanço, 1995; Tavares et al., 2000; Vitalle et al., 2003). No entanto, essa congruência deve ser vista com cautela, desde que a maioria desses estudos não identificou variáveis psicossociais, como ausência paterna e clima familiar, restringindo-se a variáveis mais socioeconômicas gerais.

Particularmente a relação entre ambiente psicossocial de criação e início da vida sexual ativa e nascimento do primeiro filho configura-se como um resultado de alto impacto para a compreensão do fenômeno, na medida em que confere a variáveis psicológicas um papel importante. Os resultados encontrados são promissores, estendendo o alcance de modelos teóricos propostos no âmbito da psicologia a uma população racial, social e culturalmente mente diversa. No entanto, o estudo representa apenas uma primeira abordagem ao problema e revelou que, dadas as características brasileiras de diversidade e miscigenação racial, e desigualdade de educação e renda, planejamentos cuidadosamente controlados quanto às variáveis relevantes são indispensáveis para testar conclusivamente as hipóteses em questão. Enquanto estudos realizados nos Estados Unidos, Europa, Austrália e Nova Zelândia têm controlado o fator racial, a situação brasileira oferece um contexto peculiar, pela grande miscigenação existente, o que, se por um lado impediria o controle da variável pela separação de grupos, por outro neutralizaria, de certa forma, essa possível influência. No entanto, a própria miscigenação brasileira não é uniforme entre as diferentes regiões e a ausência de informação sobre o assunto nesse estudo deve ser corrigida em estudos futuros. Tendo em vista também as diferenças de renda existentes no país e a existência de um grande contingente de pessoas muito pobres, é necessário controlar as variáveis relacionadas à nutrição, para permitir o estudo dos prováveis efeitos independentes de variáveis psicossociais, o que requereria estudos longitudinais. Além disso, a investigação de estressores deve ser cuidadosamente repensada para ajustar-se às características locais, incluindo arranjos familiares, violência urbana e familiar, estressores físicos como ruído e temperatura, entre outras particularidades.

\section{Referências}

Anderson, C. A., Duffy, D. L., Martin, N. G., \& Visscher, P. M. (2007). Estimation of variance components for age at menarche in twin families. Behavior Genetics, 37, 668-677. 
Belsky, J., Steinberg, L., \& Draper, P. (1991). Childhood experience, interpersonal development, and reproductive strategy: An evolutionary theory of socialization. Child Development, 62, 682-685.

Belsky, J., Steinberg, L. D., Houts, R. N., Friedman, S. L., DeHart, G., Cauffman, E., et al. (2007). Family rearing antecedents of pubertal timing. Child Development, 78(4), 1302-1321.

Blell, M., Pollard, T. M., \& Pearce, M. S. (2008). Predictors of age at menarche in the Newcastle Thousand Families Study. Journal of Biosocial Science, 40, 563-575.

Bogaert, A. F. (2008). Menarche and father absence in a national probability sample. Journal of Biosocial Science, 40, 623636.

Chisholm, J. S. (1999). Attachment and time preference. Relations between early stress and sexual behavior in a sample of American University women. Human Nature, 10, 51-83.

Danker-Hopfe, H. (1986). Menarcheal age in Europe. Yearbook of Physical Anthropology, 29, 81-112.

Draper, P., \& Harpending, H. (1982). Father absence and reproductive strategy: An evolutionary perspective. Anthropological Research, 38, 255-273.

Dunbar, J., Sheeder, J., Lezzote, D., Dabelea, D., \& StevensSimons, C. (2008). Age at menarche and first pregnancy among psychosocially at-risk adolescents. American Journal of Public Health, 98, 1822-1823.

Ellis, B. J. (2004). Timing of pubertal maturation in girls: An integrated life history approach. Psychological Bulletin, 130, 920-958.

Ellis, B. J., \& Essex, M. J. (2007). Family environments, adrenarche, and sexual maturation: A longitudinal test of a life history model. Child Development, 78, 1799-1817.

Ellis, B. J., \& Garber, J. (2000). Psychosocial antecedents of variation in girls' pubertal timing: Maternal depression, stepfather presence, and marital and familiar stress. Child Development, 71, 485-501.

Gage, T. B., MacCullough, J. M., Weitz, C. A., Dutt, J. S., \& Abelson, A. (1989). Demographic studies and human population biology. In M. A. Little \& J. D. Haas (Eds.), Human population biology (pp. 45-68). Oxford, UK: Oxford University Press.

Graber, J. A., Brooks-Gunn, J., Paikoff, R. L., \& Warren, M. P. (1994). Prediction of eating problems: An 8-year study of adolescent girls. Developmental Psychology, 30, 823-834.

Graham, M. J., Larsen, U., \& Xu, X. (1999). Secular trend in age of menarche in China: A case study of two rural counties in Anhui province. Journal of Biosocial Science, 31, 257267.

Hoier, S. (2003). Father absence and age of menarche: A test of four evolutionary models. Human Nature, 14, 209-233.

Instituto Brasileiro de Geografia e Estatística. (2009a). Censo 2000. Retrieved September 04, 2009, from http:// www.ibge.gov.br

Instituto Brasileiro de Geografia e Estatística. (2009b). PIB per capita 2003. Retrieved September 04, 2009, from http:// www.ibge.gov.br

Kanazawa, S. (2001). Why father absence might precipitate early menarche: The role of polygyny. Evolution and Human Behavior, 22, 329-334.

Kaplan, H. S., \& Gangestad, S. W. (2005). Life history theory and Evolutionary Psychology. In D. M. Buss (Ed.), The handbook of Evolutionary Psychology (pp. 68-95). Hoboken, NJ: John Wiley.
Kim, K., \& Smith, P. K. (1998). Childhood stress, behavioural symptoms and mother-daughter pubertal development. Journal of Adolescence, 21, 231-240.

Kutner, M. H., Nachtsheim, C. J., Neter, J., \& Li, W. (2005). Applied linear statistical models. New York: McGraw-Hill.

Lago, M. J. (2002). Idade da menarca, condições socioeconômicas na infância e estatura na vida adulta: $O$ estudo Pró-Saúde. Tese de Doutorado não-publicada, Instituto de Medicina Social, Universidade Federal do Rio de Janeiro, RJ.

Lordelo, E. R., França, C. B., Lopes, L. M. S., Dacal, M. P. O., Carvalho, C. S., Guirra, R. C., et al. (2006). Investimento parental e desenvolvimento da criança. Estudos de Psicologia (Natal), 11, 257-264.

Maestripieri, D., Roney, J. R., DeBias, M., Durante, K. M., \& Spaepen, G. M. (2004). Father absence, menarche and interest in infants among adolescent girls. Developmental Science, 7, 560-566.

Moffit, T. E., Caspi, A., Belsky, J., \& Silva, P. A. (1992). Childhood experience and the onset of menarche: A test of a sociobiological model. Child Development, 63, 47-58.

Núcleo de Estudos da Violência da Universidade de São Paulo \& Comissão Teotônio Vilela de Direitos Humanos. (2007). $3^{\circ}$. Relatório Nacional sobre os direitos humanos no Brasil. São Paulo, SP: Universidade de São Paulo.

Park, S. H., Shim, Y, K. Jim, H. S., \& Eun, B. L. (1999). Age and seasonal distribution of menarche in Korean girls. Journal of Adolescent Health, 25, 97.

Pettit, G. S., \& Bates, J. E. (1999). Quality of early family relationships and individual differences in the timing of pubertal maturation in girls. Journal of Personality and Social Psychology, 77, 387-340.

Picanço, M. R. A. (1995). A idade da menarca da menina brasileira: Os fatores sócio-econômicos e as diferenças regionais. Dissertação de Mestrado não-publicada, Instituto Fernandes Figueira, Rio de Janeiro, RJ.

Posner, R. B. (2006). Early menarche: A review on timing, racial differences, etiology and psychosocial consequences. Sex Roles, 54, 315-322.

Quinlan, R. J. (2003). Father absence, parental care, and female reproductive development. Evolution and Human Behavior, 24, 376-390.

Rowe, D. C. (2002). On genetic variation in menarche and age at first sexual intercourse: A critique of the Belsky-Draper hypothesis. Evolution and Human Behavior, 23, 365-372.

Rowe, D. C., \& Rodgers, J. L. (1994). A social contagion model of adolescent sexual behavior: Explaining race differences. Social Biology, 41, 1-18.

Schmitt, D. P. (2005). Sociosexuality from Argentina to Zimbabwe: A 48-nation study of sex, culture and strategies of human mating. Behavioral and Brain Sciences, 28, 247-311.

Sun, S. S., Schubert, C. M., Roche, A. F., Kulin, H. E., Lee, P. A., Himes, J. H., et al. (2002). National estimates of the timing of sexual maturation and racial differences among US children. Pediatrics, 110, 911-919.

Tavares, C. H., Haeffner, L. S., Barbieri, M. A., Bettiol, H., Barbieri, M. R., \& Souza, L. (2000). Idade da menarca em escolares de uma comunidade rural do Sudeste do Brasil. Cadernos de Saúde Pública, 16, 709-715.

Tither, J. M., \& Ellis, B. J. (2008). Impact of fathers on daughters' age at menarche: A genetically and environmentally controlled sibling study. Developmental Psychology, 44, 1409-1420. 
Lordelo, E. R., Seidl-de-Moura, M. L., Vieira, M. L., Bussab, V. S. R., Oliva, A. D., Tokumaru, R. S. \& Britto, R. C. S. (2011). Ambiente de Desenvolvimento e Início da Vida Reprodutiva em Mulheres Brasileiras.

Trivers, R. L. (1972). Parental investment and sexual selection. In B. Campbell (Ed.), Sexual selection and the descent of man, 1871-1971 (pp. 136-207). Chicago: Aldine.

Udry, J. R., \& Cliquet, R. L. (1982). The cross-cultural examination of the relationship between age at menarche, marriage and first birth. Demography, 19, 53-63.

van den Berg, S. M., \& Boomsma, D. I. (2007). The familial clustering of age at menarche in extended twin families. Behavior Genetics, 37, 661-667.

Vigil, J. M., \& Geary, D. C. (2006). Parenting and community background and variation in women's life-history development. Journal of Family Psychology, 20, 597-604.

Vitalle, M. S., Tomioka, C. Y., Juliano, Y., \& Amancio, O. M. (2003). Índice de massa corporal, desenvolvimento puberal e sua relação com a menarca. Revista da Associação Médica Brasileira, 49, 429-433.

Wu, T., Mendola, P., \& Buck, G. M. (2002). Ethnic differences in the presence of secondary sex characteristics and menarche among U.S. girls: The third national health and nutrition examination survey, 1998 - 1994. Pediatrics, 110, 752-757. 